\title{
A VARIANT OF THE CHAIN RULE FOR DIFFERENTIAL CALCULUS
}

\author{
RICHARD A. GRAFF
}

\begin{abstract}
A version of the chain rule is developed which can be applied to the construction of solutions to quasi-linear hyperbolic partial differential equations.
\end{abstract}

In this note we present a variant of the usual chain rule for differential calculus which is extremely useful in the demonstration (see [3]) that certain types of quasi-linear unbounded vector fields generate continuous flows. We consider a composition $f \circ \alpha$, where $f$ maps an open subset of a Banach space $Y$ to a Banach space $Z$, and $\alpha$ is a curve whose image is contained in the domain of $f$. By strengthening the assumptions on the differentiability of $f$, we can weaken the assumptions on $\alpha$ to something less than continuity in $Y$.

We make the following assumptions throughout this article: $X, Y$, and $Z$ are Banach spaces, with $Y$ continuously and densely included in $X ; V$ is an open subset of $Y$, and $f: V \rightarrow Z ;[a, b] \subset \mathbf{R}$, and $\alpha:[a, b] \rightarrow V . B(X, Z)$ will denote the space of continuous linear maps from $X$ to $Z, B(Y, Z)$ the space of continuous linear maps from $Y$ to $Z$. If $l \in B(Y, Z)$ has an extension to an element of $B(X, Z)$, then we will use the same symbol ("l", in this case) to denote the extension, and $\|l\|_{X, Z}$ will denote the norm of the extension.

Lemma. Let $t \in[a, b]$, and assume that $\alpha:[a, b] \rightarrow X$ is differentiable at $t$. Assume that $f$ has a Gateaux derivative at $\alpha(t)$, and that $D f(\alpha(t))$ extends to an element of $B(X, Z)$. Assume in addition that there exists $k>0$ such that $\left\|f\left(v_{2}\right)-f\left(v_{1}\right)\right\|_{Z}<$ $k\left\|v_{2}-v_{1}\right\|_{X}$ for each $v_{1}, v_{2} \in V$. Then $f \circ \alpha$ is differentiable at $t$, and $(f \circ \alpha)^{\prime}(t)=$ $D f(\alpha(t))\left(\alpha^{\prime}(t)\right)$.

Proof. Since $Y$ is dense in $X$, there exists a sequence $\left\{y_{n}\right\}_{n \in N}$ of elements of $Y$ which converges in $X$ to $\alpha^{\prime}(t)$. For each $n \in N$ and $h \neq 0$,

$$
\begin{aligned}
\| h^{-1}[f(\alpha(t+h)) & -f(\alpha(t))]-D f(\alpha(t))\left(\alpha^{\prime}(t)\right) \|_{z} \\
< & \left\|h^{-1}\left[f(\alpha(t+h))-f\left(\alpha(t)+h y_{n}\right)\right]\right\|_{z} \\
& +\left\|h^{-1}\left[f\left(\alpha(t)+h y_{n}\right)-f(\alpha(t))\right]-D f(\alpha(t))\left(y_{n}\right)\right\|_{z} \\
& +\left\|D f(\alpha(t))\left(y_{n}-\alpha^{\prime}(t)\right)\right\|_{z}
\end{aligned}
$$

Received by the editors October 4, 1979.

AMS (MOS) subject classifications (1970). Primary 58C20, $47 \mathrm{H} 99$.

Key words and phrases. Chain rule. 
Now,

$$
\begin{aligned}
& \| h^{-1}[f(\alpha(t+h))\left.-f\left(\alpha(t)+h y_{n}\right)\right]\left\|_{z}=|h|^{-1}\right\| f(\alpha(t+h))-f\left(\alpha(t)+h y_{n}\right) \|_{z} \\
&<k|h|^{-1}\left\|\alpha(t+h)-\alpha(t)-h y_{n}\right\|_{X} \\
&<k|h|^{-1}\left\|\alpha(t+h)-\alpha(t)-h \alpha^{\prime}(t)\right\|_{X}+k\left\|\alpha^{\prime}(t)-y_{n}\right\|_{X},
\end{aligned}
$$

and

$$
\left\|D f(\alpha(t))\left(y_{n}-\alpha^{\prime}(t)\right)\right\|_{z}<\|D f(\alpha(t))\|_{X, z}\left\|y_{n}-\alpha^{\prime}(t)\right\|_{X}
$$

Thus, for each $n \in N$,

$$
\begin{gathered}
\limsup _{|h| \rightarrow 0}\left\|h^{-1}[f(\alpha(t+h))-f(\alpha(t))]-D f(\alpha(t))\left(\alpha^{\prime}(t)\right)\right\|_{z} \\
<\left(k+\|D f(\alpha(t))\|_{X, z}\right)\left\|y_{n}-\alpha^{\prime}(t)\right\|_{X} .
\end{gathered}
$$

Since $\left\|y_{n}-\alpha^{\prime}(t)\right\|_{X} \rightarrow 0$ as $n \rightarrow \infty$, the lemma is proved.

Theorem. Assume that $V$ is convex, that $f$ has a Gateaux derivative at each point of $V$, that each $D f(v)$ has an extension to an element of $B(X, Z)$, and that $D f(V)$ is a bounded subset of $B(X, Z)$. Assume in addition that $\alpha(\cdot)$ is absolutely continuous and differentiable almost everywhere from $[a, b]$ to $X$. Then $f \circ \alpha$ is absolutely continuous and differentiable almost everywhere from $[a, b]$ to $Z$, and $(f \circ \alpha)^{\prime}(t)=$ $D f(\alpha(t))\left(\alpha^{\prime}(t)\right)$ for each $t$ at which $\alpha^{\prime}(t)$ exists.

Proof. Choose $c>0$ such that $\|D f(v)\|_{X, z}<c$ for every $v \in V$. From the Mean Value Theorem it follows that $\left\|f\left(v_{2}\right)-f\left(v_{1}\right)\right\|_{Z}<c\left\|v_{2}-v_{1}\right\|_{X}$ for each $v_{1}$, $v_{2} \in V$. The above lemma then implies that $(f \circ \alpha)^{\prime}(t)=D f(\alpha(t))\left(\alpha^{\prime}(t)\right)$ for each $t$ at which $\alpha^{\prime}(t)$ exists. Thus, $f \circ \alpha$ is differentiable almost everywhere. Since $\alpha(\cdot)$ is absolutely continuous from $[a, b]$ to $X$, the estimate $\left\|f\left(v_{2}\right)-f\left(v_{1}\right)\right\|_{z}<$ $c\left\|v_{2}-v_{1}\right\|_{X}$ for every $v_{1}, v_{2} \in V$ implies that $f \circ \alpha$ is absolutely continuous from $[a, b]$ to $Z$.

It is easy to use our lemma to produce versions of the above theorem in which the domain of $\alpha$ is permitted to be an open subset of an arbitrary Banach space and $\alpha$ is assumed to be Gateaux differentiable when regarded as a map into $X$ (cf. the chain rule for $\beta$-differentiability in $[1, \S 5])$. However, many such generalizations are possible and so, having no specific application in mind for such a generalization, we have chosen to restrict our attention in this note to a version of demonstrated usefulness.

The proof of our lemma is an adaptation of the proof of a weaker result which appears in [2].

\section{REFERENCES}

1. J. R. Dorroh and R. A. Graff, Integral equations in Banach spaces, a general approach to the linear Cauchy problem and applications to the nonlinear problem, J. Integral Equations 1 (1979), 309-359.

2. J. R. Dorroh and J. E. Marsden, Differentiability of nonlinear semigroups (unpublished).

3. R. A. Graff, A functional analytic approach to existence and uniqueness of solutions to some nonlinear Cauchy problems (preprint).

Department of Mathematics, Louisiana State University, Baton Rouge, Louisiana 70803 